\title{
Efficacy of nutrition interventions in ameliorating malnutrition among children: a systematic review
}

\author{
E. E. Payin ${ }^{1}$ and P. Jacob ${ }^{2}$ \\ ${ }^{1}$ Department of Food and Nutrition Education, Faculty of Home Economics Education, University of Education, \\ Winneba, Ghana and \\ ${ }^{2}$ Atlantic International University, Pioneer Plaza, Honolulu, Hawaii, USA
}

Malnutrition is a state of deficiencies, excesses, or imbalances in a person's intake of energy and or nutrients ${ }^{(1)}$. Undernutrition, micronutrient-related malnutrition and overweight, obesity and diet-related noncommunicable diseases are the three broad groups of conditions that malnutrition encompasses ${ }^{(2)}$. Around $45 \%$ of deaths among children under age 5 years are linked to Undernutrition $^{(1)}$. In 2019, globally, about 144 million children less than 5 years of age suffered from stunting, 47 million children under age 5 were wasted of which 14.3 were severely wasted and 38.3 million were overweight ${ }^{(3)}$. The goal of a nutrition intervention is to resolve or improve a nutrition diagnosis or nutrition problem by provision of advice, counselling, education, or delivery of a food component of a specific diet or meal plan tailored to a patient's needs ${ }^{(4)}$. Thus the objective of this systematic review was to evaluate the efficacy of the nutrition interventions administered for the treatment of malnutrition among children under the age of 5.

Maternal and Child Nutrition Journal, BMJ Global Health, The American Journal of Clinical Nutrition, Public Health Nutrition, and the Journal of Nutrition were searched from the Scopus and Schimago databases for this systematic review. Randomized controlled trials, cohorts, and case studies published between January 2013 and September 2020 that focused on treatment and management of malnutrition among children under the age of 5 were eligible for inclusion. The authors independently extracted the data and assessed the risk of bias of 10 included studies using Cochrane's tool for assessing the risk of bias in randomized trials.

The 13 included studies contained a sample of 45,781 under 5 children from Low and Middle-Income Countries diagnosed with severe acute, moderate acute, global acute, severe wasting, moderate wasting, and stunting. Food deliveries, such as therapeutic, supplementary, and augmented foods, nutrition counselling and education were the main interventions used. Locally developed therapeutic and supplementary ready-to-use foods were found to have similar high recovery rates from malnutrition as the standardized ones. Interventions (6/8) that combined both food delivery and either nutrition counselling or education yielded higher recovery from malnutrition. Milk, egg, and multiple micronutrient supplementations were found to improve the linear growth of +0.23 (95\% CI: $0.18,0.29 ; \mathrm{P}<0.05)$ of stunted children. About $90.3 \%$ of SAM children treated with RUTF and a high dose of vitamin D3 gained $>15 \%$ of the baseline body weight.

This review suggests that locally developed RUTFs and RUSFs can be used as cost-effective alternatives for the treatment of malnutrition among children. Nutrition education and counselling enhance the overall efficacy of nutrition interventions when included.

\section{Acknowledgements}

We wish to express our sincere thanks to the University of Education, Winneba, Ghana, and the Atlantic International University, Hawaii, USA for their support and advice towards this study.

\section{References}

1. World Health Organization (2020) [Available at:_https://www.who.int/news-room/fact-sheets/detail/malnutrition]

2. United Nations Children's Fund (UNICEF) (2009)_[Available at: https://www.unicef.org/nutrition/training/2.3/contents.html]

3. United Nations Children's Fund (UNICEF) World Health Organization, International Bank for Reconstruction and Development/The World Bank (2020) _Geneva: World Health Organization. [Available at: https://data.unicef.org/resources/jme-report-2020/

4. Academy of Nutrition and Dietetics (2014)_ [https://www.andeal.org/vault/2440/web/files/20140527-NI\%20Snapshot.pdf] 